\title{
Genomic ancestry estimation quantifies use of wild species in grape breeding
}

\author{
Zoë Migicovsky ${ }^{1}$, Jason Sawler ${ }^{1,2}$, Daniel Money ${ }^{1}$, Rudolph Eibach ${ }^{3}$, Allison J. Miller ${ }^{4}$, James J. Luby ${ }^{5}$, \\ Andrew R. Jamieson ${ }^{6}$, Dianne Velasco ${ }^{7}$, Sven von Kintzel ${ }^{8}$, John Warner ${ }^{9}$, Walter Wührer ${ }^{10}$, Patrick J. Brown ${ }^{11}$ \\ and Sean Myles ${ }^{1 *}$
}

\begin{abstract}
Background: Grapes are one of the world's most valuable crops and most are made into wine. Grapes belong to the genus Vitis, which includes over 60 inter-fertile species. The most common grape cultivars derive their entire ancestry from the species Vitis vinifera, but wild relatives have also been exploited to create hybrid cultivars, often with increased disease resistance.

Results: We evaluate the genetic ancestry of some of the most widely grown commercial hybrids from North America and Europe. Using genotyping-by-sequencing (GBS), we generated 2482 SNPs and 56 indels from 7 wild Vitis, $7 \mathrm{~V}$. vinifera, and 64 hybrid cultivars. We used a principal component analysis (PCA) based ancestry estimation procedure and verified its accuracy with both empirical and simulated data. V. vinifera ancestry ranged from $11 \%$ to $76 \%$ across hybrids studied. Approximately one third (22/64) of the hybrids have ancestry estimates consistent with F1 hybridization: they derive half of their ancestry from wild Vitis and half from $V$. vinifera.

Conclusions: Our results suggest that hybrid grape breeding is in its infancy. The distribution of $V$. vinifera ancestry across hybrids also suggests that backcrosses to wild Vitis species have been more frequent than backcrosses to $V$. vinifera during hybrid grape breeding. This pattern is unusual in crop breeding, as it is most common to repeatedly backcross to elite, or domesticated, germplasm. We anticipate our method can be extended to facilitate marker-assisted selection in order to introgress beneficial wild Vitis traits, while allowing for offspring with the highest $V$. vinifera content to be selected at the seedling stage.
\end{abstract}

\section{Background}

Grapes are one of the world's most valuable crops and although grown primarily for wine, they are also used fresh, dried and in juice [1]. In 2013, grapes had the $2^{\text {nd }}$ highest global gross production value among fruit crops, exceeded only by tomato [2]. Grapes belong to the genus Vitis, which includes over 60 inter-fertile species spread broadly across the northern hemisphere [3]. However, based on total global area in 2010, over $98 \%$ of wine grapes belong to a single species, Vitis vinifera [4]. Almost all grape cultivars grown commercially are either $V$. vinifera or hybrids that include $V$. vinifera parentage [1].

In addition to the use of one Vitis species for almost all grape growing, grapes are predominately grown using

\footnotetext{
* Correspondence: sean.myles@dal.ca

${ }^{1}$ Department of Plant and Animal Sciences, Faculty of Agriculture, Dalhousie University, Truro, NS, Canada

Full list of author information is available at the end of the article
}

vegetative propagation, which has resulted in extensive clonal relationships and limited diversity. The wine industry's preference for traditional varieties makes the acceptance of new $V$. vinifera cultivars difficult $[5,6]$. A study by Myles et al. [7] found $58 \%$ of the 950 grape cultivars examined had at least one clonal relationship. Among the unique cultivars, $74.8 \%$ had a first-degree relationship with at least one other cultivar. This extensive interrelatedness and lack of diversity have left grape cultivars susceptible to many continually evolving pathogens $[7,8]$. For example, Pierce's disease currently costs the California wine industry approximately $\$ 92$ million annually [9]. The future of the wine industry relies on the exploration of new genetic diversity through breeding.

Crop wild relatives (CWRs) provide a useful source of genetic variation for crop improvement [10-12]. An overview of 19 different crops found that more than $80 \%$ of beneficial traits from CWR genes were involved 
in pest and disease resistance [12]. By 1997, genomic crop improvements made due to CWRs had an estimated global benefit of $\$ 115$ billion annually [13]. Due to disease susceptibility of $V$. vinifera cultivars, settlers to North America had great difficulty growing the vine. These early settlers grew native, wild vines such as $V$. $l a-$ brusca and $V$. aestivalis and hybridized them with $V$. vinifera [14]. Significant exploitation of CWRs began in the 1850s when the phylloxera louse devastated European vineyards. Breeders used American wild Vitis species to develop rootstocks resistant to phylloxera, rescuing the wine industry. Commercial V. vinifera wine cultivars are still grafted onto these phylloxera-resistant rootstocks $[6,15]$.

Largely in response to the phylloxera crisis, wild Vitis were also used in scion breeding. However, the initial hybrids were generally considered undesirable for wine production due to unfavorable aromas and tastes inherited from the wild Vitis parents [16-19]. Sustained breeding enabled the development of hybrid cultivars with improved disease resistance and without the undesirable flavor compounds, including German varieties such as 'Phoenix' and 'Orion' [6]. Early French breeders, including Eugene Kuhlmann and Pierre Castel, also created well-known hybrids such as 'Marechal Foch' and 'Castel'. However, despite the promise of novel hybrid grape cultivars, their use was met with strong resistance. France introduced several wine "quality laws" prohibiting the use of many French-American hybrids $[1,20]$. French regulations influenced the perception of hybrid grape cultivars, as well as the European Union wine classification, which outlawed hybrids from the highest quality level [20].

Although it is widely believed that nearly all commercial grape varieties derive their entire ancestry from $V$. vinifera, there is increasing evidence that wild Vitis species may have been incorporated more often than previously assumed. Estimates of $V$. vinifera ancestry frequently rely on historical pedigrees from breeders, but these records may be flawed. Genomics provides a powerful tool for detecting pedigree errors and wild Vitis ancestry. For example, a recent study used nuclear microsatellite markers to determine that $33 \%$ of the 381 breeder pedigrees examined were incorrect. In most cases, the paternal parent was incorrectly identified, likely due to pollen contamination [21]. Most recently, a genomic analysis uncovered that the most important Japanese wine cultivar, 'Koshu', contained $30 \%$ wild ancestry despite being commonly classified as entirely $V$. vinifera [22].

In addition to illuminating the contribution of wild Vitis to commercial grapes, genomics can help breeders introgress desirable traits from wild relatives into new grape cultivars. Marker-assisted selection (MAS) uses genetic markers either responsible for a phenotype or strongly linked to it. MAS is especially helpful in longlived perennial crops, like grapes, where selection can be made at the seed or seedling stage, eliminating the time and money required for the plant to fully mature [23]. Moreover, combining markers linked to key traits with genomic ancestry estimates can enable breeders to select the progeny with the highest $V$. vinifera content as well as the desirable trait from the wild relative.

To enable genomics-assisted ancestry estimation in grapes, Sawler et al. [24] estimated V. vinifera ancestry in interspecific Vitis hybrids using single nucleotide polymorphism (SNP) array data from 127 accessions in the grape germplasm collection of the United States Department of Agriculture (USDA). However, the USDA collection contains relatively few commonly grown commercial hybrid cultivars. To gain insight into the ancestry across the most common commercial hybrids, we generated genotyping-by-sequencing (GBS) data and quantified $V$. vinifera ancestry from 64 of the most widely grown commercial hybrids from North America and Europe. We find that $V$. vinifera ancestry ranged from $11 \%$ to $76 \%$ across our sample of hybrid varieties. The distribution of ancestry across hybrids suggests the unusual practice of breeders backcrossing more frequently to wild Vitis species than to $V$. vinifera during hybrid grape breeding.

\section{Methods}

\section{Sample collection and genotype calling}

Leaf tissue was collected from 63 commercial grape varieties from Canada (Nova Scotia), Germany and the United States. We also used samples from $11 \mathrm{~V}$. vinifera, 6 hybrids, and 15 wild accessions from the USDA grape germplasm collection which were previously genotyped in Sawler et al. [24]. DNA was extracted using commercial extraction kits. A list of all samples is available in Additional file 1: Table S1.

A single GBS library from 96 samples was generated according to Elshire et al. [25] using two different pairs of restriction enzymes (HindIII-HF/BfaI, HindIII-HF/MseI) and was sequenced using Illumina Hi-Seq 2000 technology. Reads were aligned to the $12 \mathrm{X}$ grape reference genome from GENOSCOPE (http://www.genoscope.cns.fr/ externe/GenomeBrowser/Vitis/) using the Tassel/BWA version 5 pipeline with minimum quality score $(\mathrm{mnQS})$ of 20 and minimum kmer count (c) of 3 to generate a genotype matrix with 830,822 sites $[26,27]$. All other default parameters were used.

\section{Data curation}

VCFtools v0.1.12b [28] was used to filter for biallelic sites as well as a minimum number of reads (minDP) of 8. The file was converted into PLINK format and SNPs with $<20 \%$ missing data were retained using PLINK v1.07 $[29,30]$. Accessions with $>20 \%$ missing data were removed, followed by SNPs with a minor allele frequency 
$(\mathrm{MAF})<0.05$. SNPs with excess heterozygosity (i.e. failed a Hardy-Weinberg equilibrium test with a $p$-value $<0.001$ ) were also removed, resulting in 80 accessions and 6664 sites remaining. An identity-by-state (IBS) similarity matrix was calculated using PLINK for hybrid samples (Additional file 2: Table S2). Missing genotypes were imputed using LinkImpute [31] with optimized values of 6 and 17 for parameters $l$ and $k$, respectively, which resulted in an estimated genotype imputation accuracy of $91 \%$.

In order to perform a PCA-based admixture analysis, equal ancestral sample sizes are required [32]. We removed two random $V$. vinifera samples, and the resulting dataset contained 78 samples, which included ancestral populations of 7 wild Vitis samples and $7 \mathrm{~V}$. vinifera. Only SNPs with MAF $>0.1$ in the ancestral populations were retained across all samples.

We pruned for linkage disequilibrium using PLINK by considering a window of 10 SNPs, removing one SNP from a pair if $\mathrm{r}^{2}$ was $>0.5$ then shifting the window by 3 SNPs and repeating the procedure (PLINK command: indep-pairwise 103 0.5). 2538 sites, which included 56 indels and 2482 SNPs, remained for principal component analysis (PCA). The median distance between SNPs remaining after filtering was $1086 \mathrm{bp}$ and the inter-SNP distribution can be seen in Additional file 3: Figure S1.

\section{Ancestry estimation}

We calculated principal component (PC) axes using the ancestral $V$. vinifera and wild Vitis samples and then projected hybrid cultivars onto these axes using smartpca from the EIGENSOFT v.6.0.4 software package (Fig. 1a) [33, 34]. Based on PCA projection, ancestry coefficients for each hybrid were estimated using a similar approach described in [24, 35]. The Euclidean distance between a particular hybrid cultivar and the mean value for $V$. vinifera (a) and wild Vitis (b) populations along PC1 was calculated and the percentage of $V$. vinifera was determined using the formula ' $\%$. vinifer $=\mathrm{b} /(\mathrm{a}+\mathrm{b})^{*} 100$ ' (Fig. 1b).

\section{Simulations of admixture}

In order to determine the accuracy of the PCA-based ancestry estimates, we generated simulated offspring using data from the genotyped samples as described in Sawler et al. [24]. We estimated the proportion $V$. vinifera ancestry from simulated $\mathrm{F} 1$ hybrids, $\mathrm{F} 1 \mathrm{x} V$. vinifera backcrosses and F1 $\mathrm{x}$ wild Vitis backcrosses, which are expected to have $50 \%, 75 \%$ and $25 \% \mathrm{~V}$. vinifera ancestry, respectively. For the F1 hybrids, a parent was randomly selected from each two ancestral populations, and parental genotypes were combined by randomly sampling one allele at each site. Linkage disequilibrium between sites was ignored and the process was repeated 10,000 times in order to generate 10,000 F1 offspring. The procedure was repeated with a randomly chosen simulated $\mathrm{F} 1$ as one parent and a randomly chosen wild Vitis $(n=10,000)$ or $V$. vinifera $(n=10,000)$ as the other, in order to simulate backcrossing to the ancestral populations. The percentage $V$. vinifera ancestry and $95 \%$ confidence interval were calculated for all simulated populations.

\section{Results and discussion Method verification}

Wild Vitis species can be used in grape breeding programs to introgress disease and abiotic stress resistance
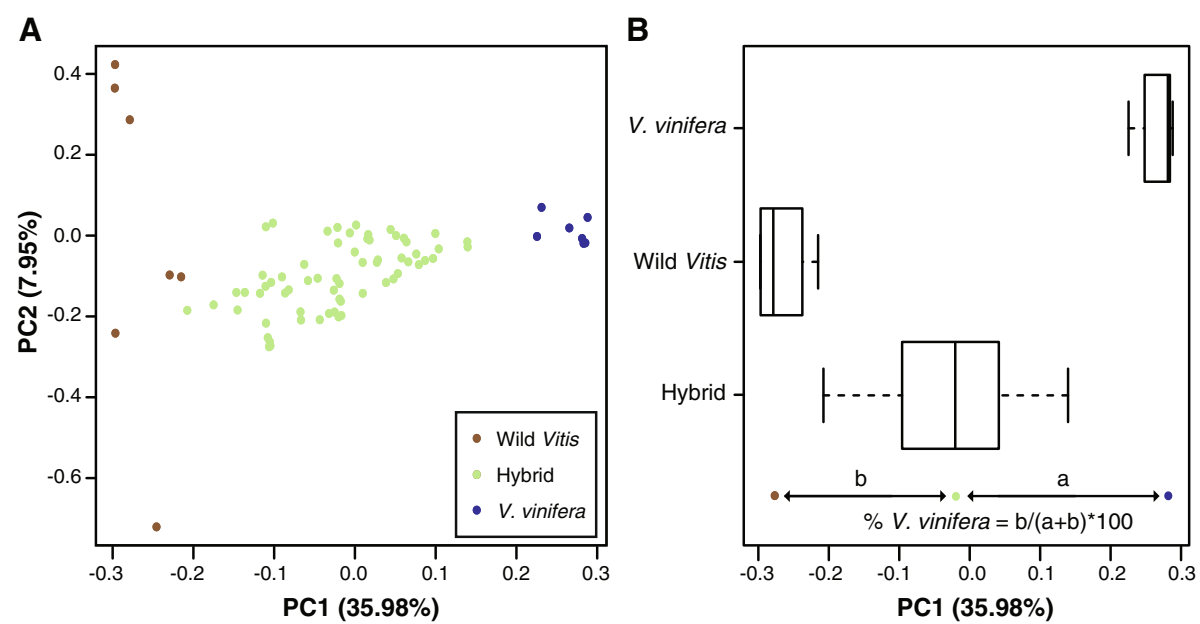

Fig. 1 PCA-based ancestry estimation using 2482 SNPs and 56 indels for 7 wild Vitis, 7 V. vinifera, and 64 hybrid samples. a PCs were generated using wild Vitis and $V$. vinifera samples. The proportion of the variance explained by each PC is shown in parentheses along each axis. Hybrids were projected onto the axes. $\mathbf{b}$ Boxplots of PC1 values for wild Vitis, V. vinifera, and hybrid cultivars as well as a visual description of the calculation used for ancestry estimation. Further details are found in the Methods 
into susceptible germplasm belonging to the domesticated grape, $V$. vinifera. Commercial cultivars with wild Vitis ancestry are often referred to as "hybrids". An evaluation of ancestry across commercial hybrids can provide insight into the history of hybrid grape breeding and a foundation for future efforts to select for ancestry based on marker data. Previous work provided accurate ancestry estimates of interspecific grape cultivars using Vitis9KSNP array data for cultivars belonging to the USDA germplasm collection [24]. We applied the same PCA-based method to evaluate the ancestry of some of the most widely grown hybrid cultivars sampled from North America and Europe using GBS data.

PCA provides a clear separation of wild Vitis and $V$. vinifera samples along $\mathrm{PC} 1$, with commercial hybrids found between the two ancestral groups (Fig. 1a). The projected position of a hybrid along PC1 was used to calculate its percentage $V$. vinifera ancestry (Fig. 1b).

In order to evaluate the accuracy of our ancestry estimates, we performed in silico crosses between wild Vitis and $V$. vinifera populations using our genome-wide SNP data to simulate F1 hybrids as well as hybrids generated from F1 simulated hybrids backcrossed to $V$. vinifera or wild Vitis. The simulated progeny were projected onto PC axes determined using the ancestral populations and the resulting PCA plot is shown in Fig. 2a.

The expected $V$. vinifera content in an F1 offspring with one $V$. vinifera and one wild Vitis parent is $50 \%$, and the mean estimated content in the simulated F1 population described here was $50.1 \%$, with a $95 \%$ confidence interval (CI) ranging from $42.7 \%$ to $57.2 \%$. In progeny produced by an F1 hybrid backcrossed to wild Vitis, the expected $V$. vinifera content is $25 \%$, which was the mean estimate of our simulated data, with a
$95 \%$ CI of $18.4 \%$ to $32.6 \%$. Finally, the mean $V$. vinifera content in simulated F1 hybrids backcrossed to $V$. vinifera is expected to be $75 \%$, and our results have a mean value of $75.1 \%$, with a $95 \%$ CI of $68.5 \%$ to $80.9 \%$. The proximity of our simulated values to expected values provides support for the accuracy of our method, but it is worth noting that our $95 \%$ confidence intervals indicate that estimates may deviate by as much as $7-8 \%$ from the expected value. Moreover, the accuracy of our estimates may decrease in cases where crosses are generated from parents whose ancestry differs significantly from the samples used as ancestral populations in the present study. Ancestry estimates for simulated progeny are shown in Fig. 2b.

\section{Commercial grape ancestry estimation}

The distribution of $V$. vinifera content estimated for the hybrid grape cultivars examined in this work is found in Fig. $3 \mathrm{a}$, and the ancestry estimates for each cultivar are listed in Fig. 3b.

Hybrids previously genotyped in Sawler et al. [24] and replicated in this study using GBS include 'Bertille-seyve 5563' (DVIT 169), 'Van Buren' (DVIT 1129), 'Rofar Vidor' (DVIT 2258), DVIT 2180, 'Jackson Sel. \#3' (DVIT 2916), and 'Marechal Foch' (California) (DVIT 214). The ancestry estimates for these samples differed by $2-5 \%$ from those previously estimated, with the exception of DVIT 2180 where our estimate of $V$. vinifera ancestry was $19 \%$ higher than in the previous work. DVIT 2180 is an unnamed accession simply identified as a Vitis species by the USDA. Given that the tissue for both studies was collected separately, the large difference in our estimates may be due to mislabelling or sample mix-up. Regardless of this discrepancy, the position of this sample
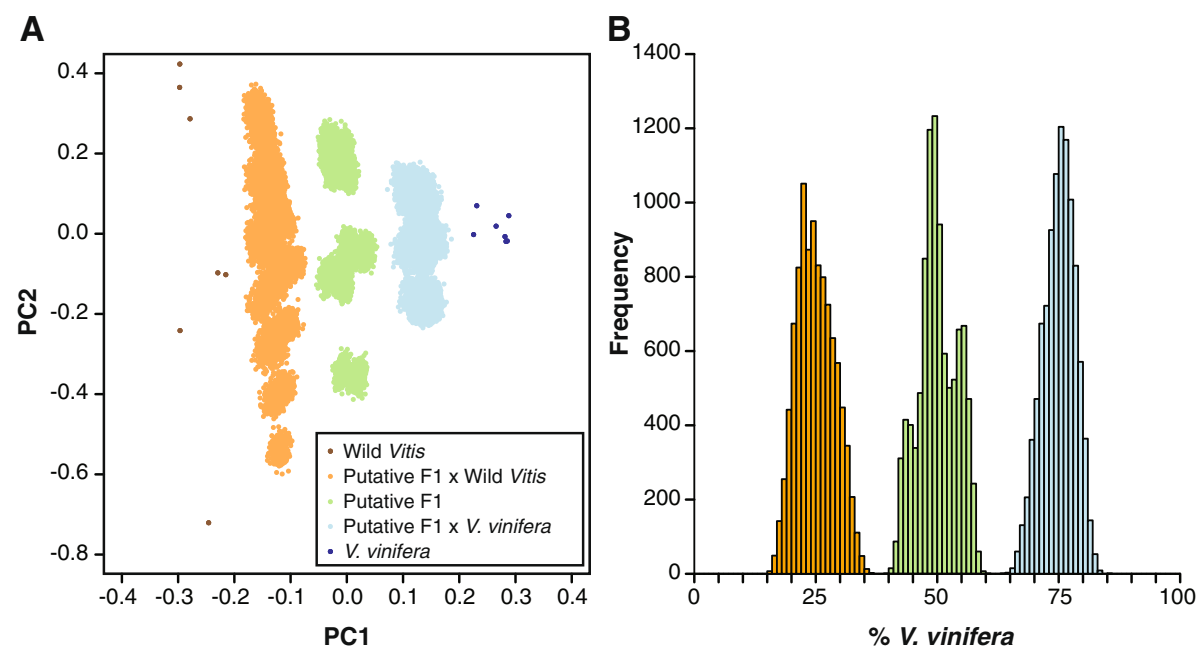

Fig. 2 Simulation of hybrids (10,000 of each). a Simulated hybrids including F1 hybrids, F1 backcrossed to V. vinifera and F1 backcrossed to wild Vitis were projected onto axes generated using wild Vitis and $V$. vinifera samples $\mathbf{b}$ Distribution of ancestry estimates for simulated populations 


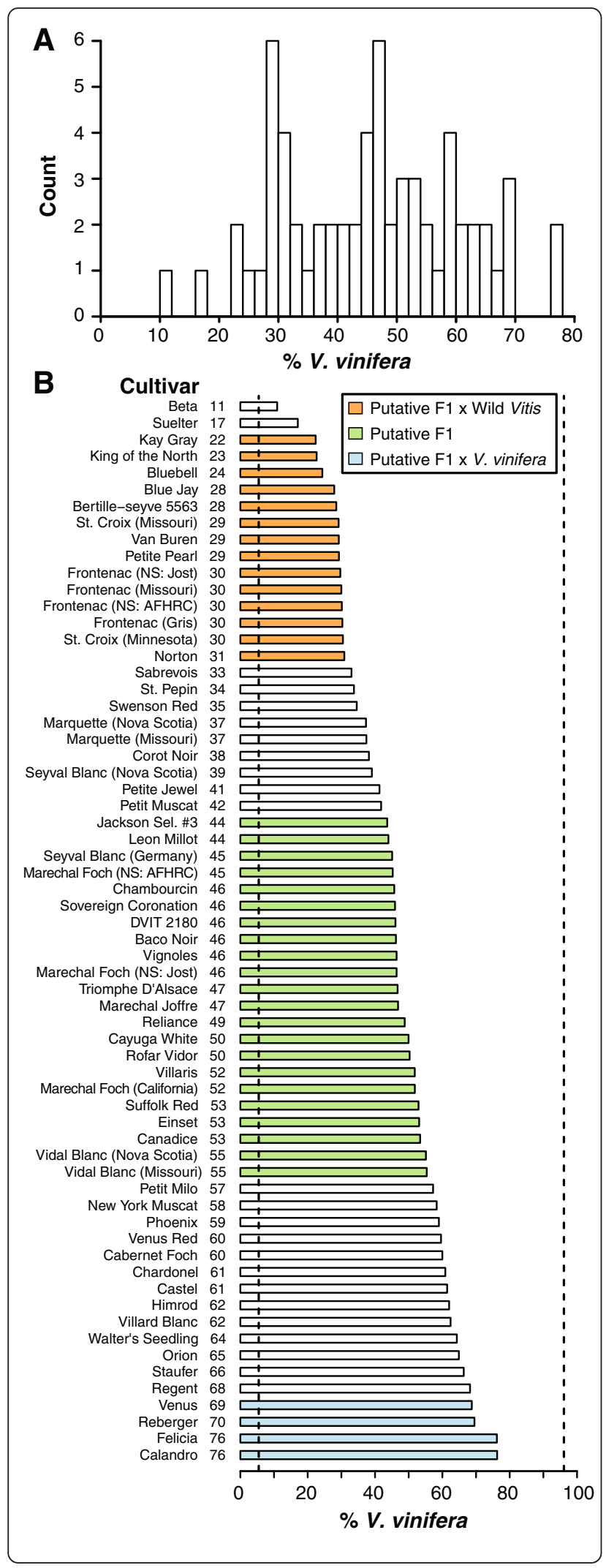

Fig. 3 Estimated V. vinifera content in 64 commercial grape hybrids. Estimates are based on 2538 sites. a Distribution of $V$. vinifera ancestry estimates in hybrids (b) $V$. vinifera ancestry estimates for each cultivar. Bars are colored if a hybrid cultivar's ancestry estimate falls within the $95 \%$ confidence interval of a F1, F1 x wild Vitis, or F1 $X V$. vinifera cross, based on simulated values. Dotted lines indicate mean values for the wild Vitis and $V$. vinifera samples

in PC space confirms that it is indeed a hybrid sample (Fig. 1a).

In order to further confirm the accuracy of our ancestry estimates, we compared $V$. vinifera ancestries inferred from well-known pedigrees to our genomicsbased ancestry estimates. For example, 'Beta' is a cross between Vitis riparia and 'Concord', a Vitis labrusca cross thought to possess some $V$. vinifera ancestry due in part to its hermaphroditic flowers [36, 37]. Sawler et al. [24] estimated the $V$. vinifera content of 'Concord' as $31 \%$. Based on these values, the percentage $V$. vinifera found in 'Beta' is expected to be approximately $16 \%$, and it was estimated as $11 \%$ here (Fig. 3a). 'Baco Noir' is a known F1 hybrid between 'Folle Blanc' ( $V$. vinifera) and $V$. riparia, and therefore it is expected to be $50 \% \mathrm{~V}$. vinifera. Our estimate is $46 \%$, which falls within the $95 \%$ confidence interval of the $V$. vinifera ancestry estimates from our simulated F1 hybrid offspring. In these two cases, our genomics-based ancestry estimates are consistent with pedigree-based estimates.

Our study also included several cultivars collected from multiple locations, and the ancestry estimates were generally similar or equivalent for these replicates from different geographic regions. For example, 'Frontenac' sampled from two locations in Nova Scotia, Missouri, as well as a Gris sport, were all estimated to be $30 \% \mathrm{~V}$. vinifera. 'Marquette' samples from both Nova Scotia and Missouri were estimated to contain $37 \% V$. vinifera. However, the ancestry estimate (52\%) for a 'Marechal Foch' accession retrieved from the USDA germplasm collection was $6 \%$ and $7 \%$ higher than the samples collected from two different locations in Nova Scotia. IBS values indicate that this sample is likely not the same cultivar as the 'Marechal Foch' grown in Nova Scotia (Additional file 4: Figure S2). Still, all ancestry estimates of 'Marechal Foch' fall within the putative F1 range, which is expected given 'Marechal Foch' is the offspring of '10114 Mgt.' (V. riparia $\mathrm{x} V$. rupestris) $\mathrm{x}$ 'Goldriesling' ( $V$. vinifera). 'Leon Millot' (44 \%) and 'Marechal Joffre' (47 \%) are siblings of 'Marechal Foch', and their ancestry estimates also fall within the range expected from an F1 hybrid (Fig. 3b) [38].

Within cultivar differences in ancestry estimates may be due partially to genotyping error. Curation error also leads to the mislabeling of samples and misidentification of cultivars. Previous work on V. vinifera cultivars from 
the USDA collection revealed widespread curation error [7], and recent work on the same collection found that the species names assigned to samples were incorrect in approximately $4 \%$ of cases [24]. In another example, three different Italian varieties all referred to as 'Bonarda' had no direct genetic relationship with each other [39]. Thus, curation error represents a likely source for the discrepancies we observe between samples with identical names.

While our data do not allow us to resolve first-degree relationships, we did examine the distribution of IBS values based on expected relationships derived from pedigree data (Additional file 4: Figure S2). We found that, while many cultivars do share alleles in a manner that supports their expected relationship, several pairs of samples that are supposed to be either geographic replicates or first-degree relatives did not have IBS values consistent with their pedigrees. For example, the IBS value for 'Villaris' and 'Felicia' (0.83) was at least 0.02 lower than all other sibling pairs examined. Additionally, the 'Seyval Blanc' sampled from Germany does not resemble the 'Seyval Blanc' from Nova Scotia to the degree we expect. In both cases, the $V$. vinifera ancestry estimates also differed. Furthermore, 'Orion', 'Staufer' and 'Phoenix' are all progeny of crosses between 'Villard Blanc' (62\%) and $V$. vinifera varieties, which has been confirmed by simple sequence repeat genotyping (Rudolf Eibach, personal communication). However, the expected ancestry for these progeny based on pedigree information should be higher $(\sim 81 \%)$ than what we observe (59\%-65\%). Further work is required in order to confirm potential sample mislabeling, cross-contamination, or genotyping error.

\section{Wild species introgression}

Often the best source for improvement of a crop plant is its wild relatives [11]. One crop that has benefited greatly from the use of wild relatives in breeding is tomato. Disease resistance in most commercial tomato cultivars is the result of genes introgressed from wild species [40, 41]. However, recurrent backcrossing to elite varieties is performed for several generations in order to remove undesirable genes introduced from the wild relative [41]. In tomato, it is customary to continue backcrossing to elite germplasm for 4 to 6 generations before the resulting hybrid is tested commercially [42].

In comparison to tomato, grape breeding appears to still be in its infancy. Approximately one third (22/64) of the hybrids analyzed in this study have $V$. vinifera content consistent with F1 hybridization (Fig. 3b). Our results suggest that grape breeders have not extensively backcrossed with $V$. vinifera in order to introgress wild genes of interest. The distribution of $V$. vinifera ancestry across hybrids actually implies that backcrosses to wild Vitis species have been more frequent than backcrosses to $V$. vinifera during hybrid grape breeding. Breeders may have generated hybrids with high wild content when aiming to introgress numerous beneficial traits from wild relatives over a small number of generations. Further local ancestry estimates would be required in order to determine the number of generations of crossing.

The high number of hybrids consistent with F1 hybridization suggests that, overall, recent hybrid grape breeding has not followed standard breeding practices that aim to introgress desirable traits from wild species by repeatedly backcrossing to elite germplasm. Alternatively, because breeders often target numerous traits for introgression from the wild, the optimal $V$. vinifera content may be lower than the desired elite content in other crops. Ultimately, the crucial factor will be which desirable parts of each ancestral genome are captured, rather than the final $V$. vinifera percentage.

One instance where repeated backcrossing to $V$. vinifera has been exploited is in the development of Pierce's disease (PD) resistant wine grapes by tracking PD resistance alleles from the wild species $V$. arizonica through MAS [43]. Seedlings resistant to PD were repeatedly backcrossed to $V$. vinifera, resulting in progeny with $97 \% V$. vinifera ancestry in the fifth generation, a value much higher than any estimates of commercial cultivars examined in this study [44]. There are many more opportunities for desirable traits, such as cold hardiness, to be introgressed from wild Vitis species into novel elite cultivars [45].

The use of molecular markers can also allow breeders to introgress multiple resistance genes into a single variety, a process called pyramiding [46]. 'Regent' is a cross between 'Diana', a $V$. vinifera variety, and the hybrid grape 'Chambourcin', which has $46 \% V$. vinifera ancestry according to our work. Based on these values, the expected $V$. vinifera ancestry of 'Regent' is approximately $73 \%$, and our estimate is $68 \%$. The complex pedigree of 'Regent' enabled the introgression of mildews and botrytis disease resistance from several Vitis species as well as high frost tolerance and early maturity [47]. In 2013, 'Regent' ranked $12^{\text {th }}$ in Germany according to total acreage [48]. Recently, 'Regent' was crossed with VHR 3082-1-42 (Muscadinia rotundifolia $\times$ V . vinifera, then backcrossed four times with $V$. vinifera) to successfully combine powdery and downy mildew resistance genes into a single variety whose ancestry likely exceeds $80 \% \mathrm{~V}$. vinifera [49].

The Institute for Grapevine Breeding Geilweilerhof, which developed 'Regent', bred 6 of the 7 cultivars with the highest $V$. vinifera content in our study (Fig. 3b). Thus, some breeders have produced hybrids with a high percentage of $V$. vinifera ancestry while retaining desirable characteristics from wild species. However, the overall lack of evidence for repeated backcrossing to $V$. vinifera in hybrid grape breeding indicates that grape breeders have yet to fully exploit the potential of combining key traits 
from wild species into novel cultivars with high $V . v i$ nifera content.

\section{Conclusions}

By examining the ancestry of 64 commercially grown grape hybrids using PCA-based ancestry estimation, we found that approximately one third of hybrids have ancestry consistent with F1 hybridization: they derive half of their ancestry from wild Vitis and half from V. vinifera, suggesting that hybrid grape breeding is in its infancy. If backcrossing to $V$. vinifera was more widely adopted, we anticipate increased acceptance of hybrid grape varieties. Improved hybrid cultivars with higher $V$. vinifera ancestry could eventually lead to the relaxation of regulations against planting hybrid grapes, and ultimately a proliferation of grape cultivars with increased abiotic and disease resistance as well as favored wine qualities.

We anticipate our method can be extended to facilitate marker-assisted selection by allowing for offspring with the highest $V$. vinifera content to be selected at the seedling stage. In combination with MAS, ancestry estimates, such as those described here, can enable the continued improvement of grape by exploiting the diversity of wild Vitis species while maintaining desirable $V$. vinifera characteristics.

\section{Additional files}

Additional file 1: Table S1. A list of the 78 cultivars used in this study as well as location and institute. Sample information is also provided for the additional 16 samples which were discarded during the genotyping analysis pipeline and they are highlighted in yellow. (XLSX $27 \mathrm{~kb}$ )

Additional file 2: Table S2. Matrix of genome-wide average IBS values for 64 hybrids. (XLSX $82 \mathrm{~kb}$ )

Additional file 3: Figure S1. Distance ( $k b)$ between filtered SNPs used for ancestry estimation. (PDF $96 \mathrm{~kb}$ )

Additional file 4: Figure S2. Distribution of IBS values for expected replicates (orange), siblings (blue) and parent/offspring (red). (A) Histogram of IBS values calculated in hybrid samples only. Dotted lines are drawn at values for expected first degree relationships as well as replicates. (B) Expected relationships between cultivars with their associated IBS values. (PDF $264 \mathrm{~kb}$ )

\section{Abbreviations}

$\mathrm{Cl}$, confidence interval; CWRs, crop wild relatives; GBS, genotyping-by-sequencing; IBS, identity-by-state; MAF, minor allele frequency; MAS, marker-assisted selection; PC, principal component; PCA, principal component analysis; PD, Pierce's disease; SNP, single nucleotide polymorphism; USDA, United States Department of Agriculture

\section{Acknowledgments}

We would like to acknowledge Bruce Reisch for useful input.

\section{Funding}

This article was written, in part, thanks to funding from the Canada Research Chairs program, the National Sciences and Engineering Research Council of Canada and Genome Canada. Z.M. was supported in part by a Killam Predoctoral Scholarship from Dalhousie University.

\section{Availability of data and material}

The dataset supporting the conclusions of this article are available in our GitHub repository at https://github.com/zoemigicovsky/grape_ancestry

\section{Authors' contributions}

Conceived and designed the experiments: ZM JS SM. Performed the experiment: ZM SM. Analyzed the data: ZM SM. Contributed reagents/materials/analysis tools: JS DM RE AJM JU ARJ DV SVK JW WW PJB. Wrote the manuscript: ZM SM. All authors have read and approved the final manuscript.

\section{Competing interests}

The authors declare that they have no competing interests.

\section{Consent for publication}

Not applicable.

\section{Ethics approval and consent to participate}

Permission was obtained to collect and use the samples within this study.

\section{Author details}

'Department of Plant and Animal Sciences, Faculty of Agriculture, Dalhousie University, Truro, NS, Canada. ${ }^{2}$ Anandia Labs, 2259 Lower Mall, Vancouver, BC, Canada. ${ }^{3} \mathrm{JKI}$ Institute for Grapevine Breeding, Geilweilerhof, Germany. ${ }^{4}$ Department of Biology, Saint Louis University, Saint Louis, MO, USA. ${ }^{5}$ Department of Horticultural Science, University of Minnesota, St. Paul, MN, USA. ${ }^{6}$ Agriculture \& Agri-Food Canada, Atlantic Food \& Horticulture Research Centre, Kentville, NS, Canada. ${ }^{~ U S D A-A R S, ~ N a t i o n a l ~ C l o n a l ~ G e r m p l a s m ~ R e p, ~}$ Davis, CA, USA. ${ }^{8}$ Jost Vineyards, 48 Vintage Lane, Malagash, NS, Canada. ${ }^{9}$ Warner Vineyards, 391 Thorpe Rd, RR\#2, Centreville, NS, Canada. ${ }^{10}$ Wührer Vineyards, 187 Highway 221, RR\#1, Kingston, NS, Canada. ${ }^{11}$ Department of Crop Sciences, University of Illinois, Urbana, IL, USA.

Received: 10 March 2016 Accepted: 13 June 2016

Published online: 30 June 2016

\section{References}

1. Reisch BI, Owens CL, Cousins PS. Grape. In: Badenes ML, Byrne DH, editors Fruit Breeding. New York, USA: Handbook of Plant Breeding: Springer US; 2012. p. 225-62.

2. Food and Agriculture Organization of the United Nations. FAOSTAT. 2015. http://faostat3.fao.org/browse/rankings/commodities_by_regions/E. Accessed October 192015.

3. This P, Lacombe T, Thomas MR. Historical origins and genetic diversity of wine grapes. Trends Genet. 2006;22(9):511-9. doi:10.1016/j.tig.2006.07.008.

4. Anderson $\mathrm{K}$, Aryal NR. A guide to where in the world various winegrape varieties are grown. Which Winegrape Varieties are Grown Where?: A Global Empirical Picture. South Australia, University of Adelaide Press; 2013. p. 1-11.

5. Bisson LF, Waterhouse AL, Ebeler SE, Walker MA, Lapsley JT. The present and future of the international wine industry. Nature. 2002:418(6898):696-9.

6. Alleweldt G, Possingham JV. Progress in grapevine breeding. Theoret Appl Genetics. 1988:75(5):669-73. doi:10.1007/BF00265585.

7. Myles S, Boyko AR, Owens CL, Brown PJ, Grassi F, Aradhya MK, et al. Genetic structure and domestication history of the grape. PNAS. 2011;108(9):3530-5.

8. Myles S. Improving fruit and wine: what does genomics have to offer? Trends Genet. 2013;29(4):190-6. doi:10.1016/j.tig.2013.01.006.

9. Alston JM, Fuller KB, Kaplan JD, Tumber KP. The economic consequences of Pierce's disease and related policy in the California winegrape industry. J Agric Resour Econ. 2013;38(2):269-97.

10. McCouch S, Baute GJ, Bradeen J, Bramel P, Bretting PK, Buckler E, et al. Agriculture: Feeding the future. Nature. 2013;499(7456):23-4. doi:10.1038/499023a.

11. Tanksley SD, McCouch SR. Seed banks and molecular maps: unlocking genetic potential from the wild. Science. 1997;277(5329):1063-6.

12. Hajjar R, Hodgkin T. The use of wild relatives in crop improvement: a survey of developments over the last 20 years. Euphytica. 2007;156(1-2):1-13. doi:10.1007/s10681-007-9363-0

13. Pimentel D, Wilson C, McCullum C, Huang R, Dwen P, Flack J, et al. Economic and environmental benefits of biodiversity. BioScience. 1997;47(11):747-57.

14. Alleweldt G. Genetics of grapevine breeding. Progress in Botany. Berlin, Heidelberg Springer; 1997. p. 441-54.

15. Zhang J, Hausmann L, Eibach R, Welter LJ, Topfer R, Zyprian EM. A framework map from grapevine V3125 (Vitis vinifera 'Schiava grossa' X 'Riesling') x rootstock cultivar 'Börner' (Vitis riparia $\times$ Vitis cinerea) to localize genetic determinants of phylloxera root resistance. Theor Appl Genet. 2009; 119(6):1039-51. doi:10.1007/s00122-009-1107-1. 
16. Narduzzi L, Stanstrup J, Mattivi F. Comparing Wild American Grapes with Vitis vinifera: A Metabolomics Study of Grape Composition. J Agric Food Chem. 2015;63(30):6823-34. doi:10.1021/acs.jafc.5b01999.

17. Sun Q, Gates MJ, Lavin EH, Acree TE, Sacks GL. Comparison of Odor-Active Compounds in Grapes and Wines from Vitis vinifera and Non-Foxy American Grape Species. J Agric Food Chem. 2011;59(19):10657-64. doi:10.1021/jf2026204.

18. Liang Z, Wu B, Fan P, Yang C, Duan W, Zheng X, et al. Anthocyanin composition and content in grape berry skin in Vitis germplasm. Food Chem. 2008;111(4):837-44. doi:10.1016/j.foodchem.2008.04.069.

19. Liang Z, Yang Y, Cheng L, Zhong G-Y. Polyphenolic composition and content in the ripe berries of wild Vitis species. Food Chem. 2012;132(2): 730-8. doi:10.1016/j.foodchem.2011.11.009.

20. Meloni G, Swinnen J. The Political Economy of European Wine Regulations. J Wine Econ. 2014;8(03):244-84. doi:10.1017/jwe.2013.33.

21. Lacombe T, Boursiquot JM, Laucou V, Di Vecchi-Staraz M, Peros JP, This P. Largescale parentage analysis in an extended set of grapevine cultivars (Vitis vinifera L.). Theor Appl Genet. 2013;126(2):401-14. doi:10.1007/s00122-012-1988-2.

22. Goto-Yamamoto N, Sawler J, Myles S. Genetic Analysis of East Asian Grape Cultivars Suggests Hybridization with Wild Vitis. PloS One. 2015;10(10): e0140841. doi:10.1371/journal.pone.0140841.

23. McClure KA, Sawler J, Gardner KM, Money D, Myles S. Genomics: a potential panacea for the perennial problem. Am J Bot. 2014;101(10):1780-90. doi:10.3732/ajb.1400143.

24. Sawler J, Reisch B, Aradhya MK, Prins B, Zhong GY, Schwaninger H, et al. Genomics assisted ancestry deconvolution in grape. PloS One. 2013;8(11): e80791. doi:10.1371/journal.pone.0080791.

25. Elshire RJ, Glaubitz JC, Sun Q, Poland JA, Kawamoto K, Buckler ES, et al. A robust, simple genotyping-by-sequencing (GBS) approach for high diversity species. PloS One. 2011;6(5):e19379. doi:10.1371/journal.pone.0019379.

26. Li H, Durbin R. Fast and accurate short read alignment with BurrowsWheeler transform. Bioinformatics. 2009;25(14):1754-60. doi:10.1093/ bioinformatics/btp324

27. Glaubitz JC, Casstevens TM, Lu F, Harriman J, Elshire RJ, Sun Q, et al. TASSELGBS: A High Capacity Genotyping by Sequencing Analysis Pipeline. PloS One. 2014;9(2):e90346. doi:10.1371/journal.pone.0090346.

28. Danecek P, Auton A, Abecasis G, Albers CA, Banks E, DePristo MA, et al. The variant call format and VCFtools. Bioinformatics. 2011;27(15):2156-8. doi:10.1093/bioinformatics/btr330.

29. Purcell S. PLINK v.1.072009. http://pngu.mgh.harvard.edu/purcell/plink/.

30. Purcell S, Neale B, Todd-Brown K, Thomas L, Ferreira MAR, Bender D, et al. PLINK: A Tool Set for Whole-Genome Association and Population-Based Linkage Analyses. Am J Hum Genet. 2007;81(3):559-75. doi:10.1086/519795.

31. Money D, Gardner K, Migicovsky Z, Schwaninger H, Zhong GY, Myles S. Linklmpute: Fast and Accurate Genotype Imputation for Non-Model Organisms. G3. 2015;5(11):23383-2390. doi:10.1534/g3.115.021667.

32. McVean G. A genealogical interpretation of principal components analysis. PLoS Genet. 2009:5(10):e1000686. doi:10.1371/journal.pgen.1000686.

33. Patterson N, Price AL, Reich D. Population structure and eigenanalysis. PLoS Genet. 2006;2(12):e190. doi:10.1371/journal.pgen.0020190.

34. Price AL, Patterson NJ, Plenge RM, Weinblatt ME, Shadick NA, Reich D. Principal components analysis corrects for stratification in genome-wide association studies. Nat Genet. 2006;38(8):904-9. doi:10.1038/ng1847.

35. Bryc K, Auton A, Nelson MR, Oksenberg JR, Hauser SL, Williams S, et al. Genome-wide patterns of population structure and admixture in West Africans and African Americans. Proc Natl Acad Sci USA. 2010;107(2):786-91. doi:10.1073/pnas.0909559107.

36. Swenson EP. Wild Vitis riparia from northern US and Canada-breeding source for winter hardiness in cultivated grapes-a background of the Swenson hybrids. Fruit Varieties J. 1985;39:28-31.

37. Cahoon C. The Concord Grapes. Fruit Varieties J. 1986;40(4):106-7.

38. Pollefeys $P$, Bousquet J. Molecular genetic diversity of the French-American grapevine hybrids cultivated in North America. Genome. 2003;46(6):1037-48. doi:10.1139/g03-076

39. Martínez L, Cavagnaro P, Boursiquot J-M, Agüero C. Molecular characterization of Bonarda-type grapevine (Vitis vinifera L.) cultivars from Argentina, Italy, and France. Am J Enology Viticulture. 2008;59(3):287-91.

40. Foolad MR. Genome mapping and molecular breeding of tomato. Int J Plant Genomics. 2007;2007:64358. doi:10.1155/2007/64358.

41. Menda N, Strickler SR, Edwards JD, Bombarely A, Dunham DM, Martin GB, et al. Analysis of wild-species introgressions in tomato inbreds uncovers ancestral origins. BMC Plant Biol. 2014;14(1):287.
42. Bai $Y$, Lindhout P. Domestication and breeding of tomatoes: what have we gained and what can we gain in the future? Ann Bot. 2007;100(5):1085-94. doi:10.1093/aob/mcm150.

43. Riaz S, Tenscher AC, Graziani R, Krivanek AF, Ramming DW, Walker MA. Using marker-assisted selection to breed Pierce's disease-resistant grapes. A Enology Viticulture. 2009;60(2):199-207.

44. Walker MA, Riaz S, Tenscher A. Optimizing the Breeding of Pierce's Disease Resistant Winegrapes with Marker-Assisted Selection. International Society for Horticultural Science (ISHS). Leuven, Belgium: Acta Hortic; 2014.

45. Zhang J, Wu X, Niu R, Liu Y, Liu N, Xu W, et al. Cold-resistance evaluation in 25 wild grape species. VITIS J Grapevine Res. 2015:51(4):153.

46. Joshi RK, Nayak S. Gene pyramiding-A broad spectrum technique for developing durable stress resistance in crops. Biotechnol Mol Biol Rev. 2010; 5(3):51-60.

47. Eibach $\mathrm{R}$, Töpher $\mathrm{R}$, editors. Success in resistance breeding: "Regent" and its steps into the market. VIII International Conference on Grape Genetics and Breeding 603. Kecskemet, Hungary, Acta Horticulturae; 2002.

48. Ruehl E, Schmid J, Eibach R, Töpfer R. Grapevine breeding programmes in Germany. In: Reynolds A, editor. Grapevine Breeding Programs for the Wine Industry. Oxford: Woodhead Publishing; 2015. p. 77-101.

49. Eibach R, Zyprian E, Welter L, Töpfer R. The use of molecular markers for pyramiding resistance genes in grapevine breeding. VITIS J Grapevine Res. 2007;46(2):120-4.

\section{Submit your next manuscript to BioMed Central and we will help you at every step:}

- We accept pre-submission inquiries

- Our selector tool helps you to find the most relevant journal

- We provide round the clock customer support

- Convenient online submission

- Thorough peer review

- Inclusion in PubMed and all major indexing services

- Maximum visibility for your research

Submit your manuscript at www.biomedcentral.com/submit
) Biomed Central 